\title{
Les heures du crépuscule de l'école de Lausanne
}

\section{Giovanni Busino}

\section{OpenEdition}

\section{Journals}

Édition électronique

URL : http://journals.openedition.org/ress/753

DOI : $10.4000 /$ ress.753

ISSN : 1663-4446

\section{Éditeur}

Librairie Droz

\section{Édition imprimée}

Date de publication : 1 juillet 2010

Pagination : 45-60

ISBN : 978-2-600-01449-6

ISSN : 0048-8046

Référence électronique

Giovanni Busino, "Les heures du crépuscule de l'école de Lausanne », Revue européenne des sciences sociales [En ligne], XLVIII-146 | 2010, mis en ligne le 01 juillet 2013, consulté le 02 mai 2019. URL http://journals.openedition.org/ress/753 ; DOI : 10.4000/ress.753 


\section{Giovanni BUSINO}

\section{LES HEURES DU CRÉPUSCULE DE L'ÉCOLE DE LAUSANNE}

L'Université de Lausanne, pendant environ cinq lustres, a eu deux enseignants, Léon Walras et Vilfredo Pareto, à la renommée mondiale. Ils ont eu des disciples, des commentateurs, des traducteurs, des vulgarisateurs dans tous les pays de la planète. Toujours regardés comme les pierres angulaires de l'Ecole de Lausanne ${ }^{1}$, ces deux savants n'ont jamais eu des disciples ou des continuateurs en terre vaudoise. Même les éditions scientifiques de leurs travaux ont été programmées et élaborées ailleurs par des chercheurs étrangers.

Afin d'expliquer ce paradoxe il faudrait connaître préalablement la sociologie historique du mode de production et de reproduction du milieu universitaire vaudois, des temps de la fondation de l'Académie à la création de l'Université, les mécanismes et le fonctionnement assurant l'égalitarisme du pouvoir collégial dans les instances académiques. Est-ce que la formation d'écoles de pensée et l'émergence de disciples adhérant ou professant les doctrines d'un maître seraient perçues, peut-être inconsciemment, comme des facteurs favorisant l'éclosion de distinctions entre les enseignants et par conséquent comme un péril pour le modèle de fonctionnement égalitariste de l'institution? Ou bien est-ce avant tout l'impératif de fonctionner, au moins jusqu'au commencement du Rectorat de Dominique Rivier, en tant qu'institution d'enseignement supérieur chargée de former surtout les élites professionnelles du Canton de Vaud?

Les réponses à des telles questions exigent des connaissances ponctuelles sur les caractéristiques socio-anthropologiques de la culture universitaire vaudoise. Ces connaissances, pour le moment, nous font défaut. En attendant, il ne nous reste qu'à parcourir les informations grappillées dans les travaux des historiens et dans les documents publiés par les archivistes. Sur le remplacement de Pareto économiste il y a déjà une recherche bien documentée ${ }^{2}$. Des sources éparses et disparates, fournissent d'autres informations à propos de son remplacement en tant qu'enseignant de sociologie.

1 Depuis quelques temps on a commencé à souligner les différences méthodologiques et épistémologiques entre Walras et Pareto ainsi que la discontinuité entre leurs deux programmes scientifiques. Pour cette raison P. Bridel \& F. Mornati, De l'équilibre général comme «branche de la métaphysique» ou de l'opinion de Pareto sur le projet walrasien, «Revue économique», vol. 60, 2009, n. 4, pp. 869-890, demandent: «[...] peut-on sérieusement parler d'une "Ecole de Lausanne" si la convergence de vue entre ses deux grands fondateurs se borne à l'adoption d'instruments analytiques commun?». Une telle question mérite une étude approfondie. La thèse d'une antithèse considérable entre les théories de Walras et celles de Pareto a été avancée en 1969 par le Prix Nobel G. J. Stiegler. Son article «È utile il passato in economia?» se trouve actuellement dans le volume du même Mercato, Informazione, regolamentazione, Bologna, Il Mulino, 1994.

2 F. Mornati, Pasquale Boninsegni e la Scuola di Losanna, Torino, Utet, 1999. 
Il est notoire que dès son arrivée à Lausanne, en 1893, Pareto devient ami du romaniste Henri Erman et du juriste et philosophe du droit Ernest Roguin, dont il appréciait surtout les polémiques contre le droit naturel. Grâce à Roguin, il fait la connaissance d'Adrien Naville et plus tard d'André Mercier, collègues et amis avec lesquels les échanges intellectuels seront constants et fructueux ${ }^{3}$.

La rencontre avec Maurice Millioud advient, en 1897, lorsque celui-ci est nommé professeur de philosophie à la Faculté des Lettres et qu'il ouvre sa leçon inaugurale par la citation parétienne: «L'esprit humain est impuissant à atteindre toute vérité dernière ». Pareto avait lu la thèse de Millioud d'Ernest Renan sur la religion, ainsi que son étude sur les théories de l'Ecole naturaliste, mais l'approche «apologétique» de ces deux travaux n'avait suscité en lui, lecteur assidu des écrivains naturalistes et de Renan, aucun intérêt. Le livre sur le système philosophique de Schopenhauer et l'essai sur le pessimisme lui paraissaient d'une facture trop spéculative. Il était sceptique à propos de la croyance affichée par Millioud selon laquelle l'activité créatrice, les «forces morales de la conscience, de la vie, du progrès », font l'histoire car il était persuadé que la science ne peut rien dire de fondé sur des telles questions. Convaincu que la raison est inapte à faire émerger l'action politique, Pareto attachait une grande importance aux sentiments, aux relations subjectives, à la «logique du non logique» des actions. En revanche, les articles de Millioud sur des problèmes d'actualité et de société paraissant dans divers quotidiens romands, Pareto les jugeaient pertinents et bien argumentés ${ }^{4}$.

Assez vite les échanges intellectuels entre les deux hommes deviennent réguliers et cordiaux. Les contributions de Millioud à propos de la réorganisation des systèmes d'enseignement ${ }^{5}$ sont très appréciées, ainsi que les essais sur la formation de la personnalité, sur l'histoire naturelle des idées et leur propagation ${ }^{6}$.

3 G. Busino, Contributi alla storia del pensiero politico contemporaneo. I. Ernest Roguin e Vilfredo Pareto, «Cahiers Vilfredo Pareto», 1964, n. 4, pp. 189-210 et Ricerche sulla diffusione delle dottrine della Scuola di Losanna, «Cahiers Vilfredo Pareto», n. 22-23, 1970, pp. 243-262. Mais aussi G. Busino et S. Stelling-Michaud, Matériaux pour une histoire des sciences sociales, «Cahiers Vilfredo Pareto», 1965, n. 6, passim et G. Busino, Recherches préparatoires à une histoire de l'épistémologie des sciences humaines, «Revue européenne des sciences sociales», XLIII, 2005, n. 132, pp. 109-140.

4 Voir à ce propos D. Christoff, in La philosophie dans la Haute Ecole de Lausanne, 1542-1955, Lausanne, Université, 1987, pp. 76-80, et M. Zürcher, Unterbrochene Tradition. Die Anfänge des Soziologie in der Schweiz, Zürich, Chronos Verlag, 1995, pp. 53-59, et du même La reconstruction d'une tradition oubliée: les débuts précoces de la sociologie en Suisse, «Les annuelles», 1997, n. 8, pp. 109-133.

5 M. Millioud, De l'enseignement de la morale à l'Ecole industrielle cantonale. Rapport présenté à la Conférence des maîtres, Lausanne, Borgeaud, 1900; L'enseignement à l'exposition universelle, Lausanne, Payot, 1901; L'enseignement supérieur, Viret-Genton, 1901; La réforme de l'enseignement secondaire dans le Canton de Vaud, S.1.S.n. 1903. Millioud a joué un rôle important dans la création du Séminaire pédagogique vaudois chargé de la formation aux professions enseignantes. Sur son rôle au moment de la création de ce Séminaire d'autres informations se trouvent in D. Hameline, Profil d'éducateurs: Edouard Claparède (1873-1940), «Perspective», XVI, 1986, n. 3, pp. 437-444 et XXIII, 1993, n.1-2, pp. 161-173; R. Hofstetter et B. Schneuwly, avec la collaboration de V. Lussi, Emergence des sciences de l'éducation en Suisse à la croisée de traditions académiques contrastées/fin du $19^{e}$-première moitié du $20^{e}$ siècle, Berne, Lang, 2007; J.-C. Bussard, L'éducation physique suisse en quête d'identité (1800-1930), Paris, L'Harmattan, 2007.

6 M. Millioud, Essai sur l'histoire naturelle des idées, «Revue philosophique», XXXIII, 1908, n. 2, pp. 113-144 et La propagation des idées, «Revue philosophique», XXXVI, 1910, n. 8, pp.143-152. 
Le 2 décembre 1901, Pareto et ses collègues de la Faculté de droit proposent à l'Université la création d'un cursus en sciences sociales. Une commission, composée de V. Pareto, E. Roguin, M. Millioud et E. Rossier, est alors chargée d'élaborer le règlement et le plan d'études de la nouvelle filière. Les travaux de la commission se prolongent pendant un semestre. Dans son rapport, la commission se borne à proposer quelques cours nouveaux à ajouter aux enseignements déjà existants en Droit et en Lettres. Pareto avance des réserves sur le programme de la sociologie «à la française» et déclare: «[...] je n'ai pas en grande estime les Durkheim, Worms et C.ie. Ce qui manque précisément à ces messieurs, c'est la connaissance de l'économie politique et des méthodes rigoureusement scientifique $»^{7}$. Le 25 juin 1902, l'Université approuve le règlement et le plan d'études, aussitôt ratifiés par le Département cantonale de l'Instruction publique.

Après avoir révisé le libéralisme classique et mis l'accent sur l'hégémonie des intérêts et des sentiments dans les choix humains et dans l'action sociale, Pareto, dans Les systèmes socialistes, présente une première formulation de la théorie des aristocraties/élites. Millioud lui manifeste des réserves assez vagues à propos de la conceptualisation de la problématique et de la définition de la notion d'élite adoptées. Aussitôt Pareto riposte: "Il est évident que nous donnons un sens différent au terme élite. [...] il peut y avoir une élite de brigands comme une élite de saints. L'élite politique n'est pas la moins du monde une élite morale ni même intellectuelle ${ }^{8}$. Néanmoins leurs échanges intellectuels se poursuivent sans d'autres anicroches. Mais ce sont surtout les sujets politiques et les questions de société qui demeurent au centre de leurs rencontres, de leurs lettres, de leurs débats.

Quand la santé l'oblige à renoncer à l'enseignement, Pareto n'a aucune hésitation à proposer Millioud comme son remplaçant. Il écrira aux autorités universitaires: «Nous avons la chance d'avoir, en notre collègue Millioud, l'homme qui peut donner un enseignement vraiment scientifique de la sociologie. Cette chance, il ne faudrait pas la négliger et la laisser échapper $»^{9}$. Cependant Millioud hésite, malgré les sollicitations pressantes. Pareto insiste, l'encourage à accepter la charge et pour cette raison lui écrit une lettre dans laquelle on peut lire: «Personne mieux que vous pourrait faire ce cours. Je ne puis pas vous donner grand espoir que ma santé ne se rétablisse jamais; mais enfin, si cela avait lieu, je serais heureux de partager avec vous le cours de sociologie, et d'en reprendre une petite partie. Je ne connais personne pouvant faire actuellement un cours de sociologie scientifique à notre Université; vous seul pouvez vous en charger. Je fais donc des vœux pour que vous vous décidiez à faire ce sacrifice. Vous avez la force qui me manque; vous ne devez pas laisser dans l'embarras notre Faculté de sciences sociales $»^{10}$.

${ }^{7}$ Lettre du 12 décembre 1901 à M. Millioud in Fondo Vilfredo Pareto della Banca Popolare di Sondrio-IT PopSo FP R11C367.

${ }^{8}$ Lettre du 5 novembre 1902 à M. Millioud in Fondo Vilfredo Pareto della Banca Popolare di Sondrio-IT PopSo FP R12C240.

9 V. Pareto, Euvres complètes (=O.C.), 19, p. 709, lettre du 21 septembre 1910.

${ }^{10}$ Lettre de V. Pareto à M. Millioud du 27 septembre 1910 in Fondo Vilfredo Pareto della Banca Popolare di Sondrio-IT PopSo FP R 16CO18. 
Enfin, Millioud accepte de se charger de l'enseignement de la sociologie, tandis que Pareto obtient un congé maladie et garde le statut de professeur de sciences sociales et politiques et la possibilité de donner, dès que sa santé le lui permettra, des cours temporaires ${ }^{11}$. Cela va être à l'origine de tous les malentendus et des incompréhensions avec Millioud.

Des témoignages rapportent qu'au fil de ses leçons, le nouvel enseignant analyse, sans dogmatisme, les théories sociologiques alors en vogue, qu'il formule quelques réserves critiques de nature formelle sans toutefois jamais prendre parti. Les auditeurs percevaient cette attitude tantôt comme une manifestation de scepticisme, tantôt comme un dilettantisme paradoxal. Le cahier des notes d'un étudiant semble confirmer que les premiers cours professés étaient des exposés et des commentaires élogieux des doctrines parétiennes ${ }^{12}$. Les références aux livres des sociologues alors en vogue sont rares et rhapsodiques.

Vers 1909 Pareto fait la connaissance d'un jeune étudiant, candidat à la licence et au doctorat en droit, lequel lui soumet un projet de thèse sur l'emploi des mathématiques en économie. Après quelques hésitations, Pareto, en janvier 1910, tout en reconnaissant l'importance du sujet, exprime ses doutes sur la possibilité de le traiter convenablement «sans avoir des connaissances mathématiques étendues $\gg^{13}$. Après des explications jugées satisfaisantes, Pareto accepte la direction de cette thèse et rencontre Pierre Boven le 14 mars $1910^{14}$. Les rapports avec le professeur deviennent réguliers et, en peu de temps, même très cordiaux. Boven, pendant le mois de juillet de 1910, est chargé de la correction des épreuves du livre Le mythe vertuiste et la littérature immorale ${ }^{15}$. En août il décide d'aller en Italie pour apprendre l'italien et prendre connaissance des travaux des historiens des doctrines économiques. Pareto en parle à Maurice Millioud et celui-ci promet de «vous donner des bons conseils » ${ }^{16}$. Boven part pour Florence d'où il informe le professeur sur l'état de ses recherches, mais aussi sur ce qu'il lui arrive de lire dans la presse locale ${ }^{17}$. Pareto demande à Guido Sensini d'aider « un mio studente che sta scrivendo una tesi sulla storia dell' economia pura» et, plus tard, il l'introduit auprès de Stanislao Scalfati ${ }^{18}$. Rentré à Lausanne à la fin du mois d'octobre de 1910, Boven repart pour Florence aux débuts de mars 1911. En juin, Pareto lui demande des nouvelles sur l'état d'avancement de ses recherches («J'espère que votre thèse avance»). En août, il se dit «heureux d'apprendre que votre thèse est à bon port, Je pense que vous pourrez ainsi bientôt avoir votre soutenance », et aux

${ }^{11}$ G. Busino et P. Bridel, L’Ecole de Lausanne de Léon Walras à Pasquale Boninsegni, Lausanne, Université, 1987, pp. 54-66.

${ }^{12}$ En voir la liste in V. Pareto, O.C., 32, pp. 325-335.

${ }^{13}$ V. Pareto, O.C., 19, p. 688, lettre du 26 janvier 1910.

14 V. Pareto, O.C., 19, pp. 690-691.

15 V. Pareto, O.C., 19, pp. 701-702, lettre du 22 juillet 1910. Cette collaboration est formalisée en octobre, O.C., 19, pp. 710-711, lettres du 6 et 13 octobre 1910.

16 V. Pareto, O.C., 19, pp. 702-703.

17 V. Pareto, O.C., 19, p. 707, lettre du 11 septembre 1910: «J'ai vu l'article dont vous me parlez. Il m'a amusé et instruit; entre autre chose j'ai appris que j'étais socialiste. Cela m'a fait un sensible plaisir.»

${ }^{18}$ V. Pareto, O.C., 19, p. 704, lettre du 28 août 1910, p. 704 et lettre de 17 décembre 1911, p. 753. 
débuts de septembre il ajoute: «Je suppose que vous aurez votre soutenance à l'automne prochain». Pendant les mois de septembre, octobre, novembre et décembre, Pareto examine le manuscrit, prépare «les observations qu'éventuellement je pourrais avoir à faire » et demande de préciser mieux un certain nombre de concepts, tels que ceux de concurrence et d'ophélimité ${ }^{19}$.

La maladie empêche le directeur de thèse d'assister à la soutenance, jugée par les membres de la commission de soutenance «brillante». Pareto s'empresse de demander à ses amis de faire paraître, dans les revues d'économie, des comptes rendus du travail ${ }^{20}$ et lui-même en publie un dans la revue anglaise Economic Journal ${ }^{21}$.

Les conditions de sa santé lui empêchant aussi de faire partie du jury de la thèse de Maria Kolabinska, La circulation des élites en France ${ }^{22}$, Pareto confie à Boven la tâche de le représenter à la soutenance et d'assurer la défense de ses théories, «la défense de la science expérimentale contre les foudres métaphysiques de Monsieur de Tourtelon et $\mathrm{Cie} »^{23}$. Il le charge également de lire une lettre, pendant la soutenance, par laquelle il manifeste son opinion sur le travail de la candidate au doctorat et sa crainte que les critiques du jury ne s'adressent, en réalité, non à la candidate mais à lui, auteur de la théorie des élites.

Au cours de la soutenance du 10 mai 1912, les critiques de l'utilisation des approches méthodologiques parétiennes par la candidate sont nombreuses. Millioud conclut son intervention en affirmant «que la candidate aurait mieux fait de ne pas entreprendre ce travail». Boven et Scalfati réagissent avec mesure et rappellent que, pour Pareto, la circulation des élites n'explique pas tout, qu'elle n'est pas la seule cause des phénomènes sociaux, qu'il n'y a pas un principe primordial, une cause unique susceptible d'expliquer l'interdépendance des phénomènes économiques et sociaux, et que la candidate a simplement rendu compte de ces éléments.

19 V. Pareto, O.C., pp. 707, 710-711, 712, 721, 728, 732, 737, 740, 744-745, 747.

${ }^{20}$ V. Pareto, O.C., pp. 760, 761, 763-764. Le titre de la thèse est: Les applications mathématiques à l'économie politique, Lausanne, Pache-Varidel \& Bron, 1912. Les comptes rendus ont été nombreux, in «Bulletin financier suisse», 17 août 1912, pp. 213-214; «La libertà economica», n. 13-14, 25 maggio 1912; «Giornale degli Economisti e Rivista di statistica», giugno 1912; «La Riforma sociale », luglio-agosto-settembre 1912; «Rivista italiana di sociologia», XVI, 1912, fasc. III-IV; «Le génie civil», LXI, 1912, n. 24; «L'Economista», $1^{\text {er }}$ décembre 1912; «L'Italia finanziaria», 3 avril 1913. Tous ces comptes rendus soulignent «la puissance d'une documentation judicieusement sélectionnée », l'utilité des mathématiques pour l'économie sans pour autant lui attribuer une importance exceptionnelle pour le développement des théories économiques. Pareto cite le travail de Boven in O.C., 9, p. 164 et in O.C., 16, pp. 181-182. Il écrira à Maffeo Pantaleoni le 19 mars 1912, O.C., 28-3, pp. 165-164: «Avrai ricevuto un libro di Boven sull'economia matematica. È ottimo, ed esprime benissimo le differenze tra le altre teorie e la mia». Le 18 avril 1912 lui écrira à nouveau, O.C., 28-3, p. 165 : le livre «mi pare ottimo».

${ }^{21}$ «Economic Journal», 1912, pp. 467-469 et puis en anglais in O.C., 16, pp. 181-182 et en français in O.C., 32, pp. 222-223.

${ }^{22}$ R.A. Murray dans le compte rendu qu'il publia dans la «Rivista italiana di sociologia», XVI, 1912, fasc. III-IV, écrira que l'auteure s'était bornée à reprendre tel quel les thèses de Pareto sur la circulation des élites.

${ }^{23}$ V. Pareto, O.C., 19, p. 765, lettre du $1^{\text {er }}$ avril 1912, ainsi que les lettres du 4, 8 et 27 avril, in O.C., 19 , pp. $765-766,766$ et 767 . 
Le procès-verbal, rédigé par Millioud, rapporte les tensions et les affrontements de cette séance académique un peu tumultueuse, inhabituellement désordonnée. D'après ce document, Boninsegni menace d'abandonner la séance tandis que Roguin et Sirven tentent vainement de ramener le calme ${ }^{24}$. Millioud est à ce point fâché et affecté par le déroulement de la soutenance que, le jour suivant, il renvoie à Pareto, sans un mot d'explication, le manuscrit du Trattato di sociologia generale qu'il avait commencé à traduire en français. Le 15 mai 1912, Pareto lui écrit: «Je viens de recevoir un paquet contenant le Ve chapitre de ma Sociologie. Je n'y ai trouvé aucune lettre de vous; ce qui fait que j'ignore pourquoi vous me renvoyez ce manuscrit. Je vous serais fort reconnaissant de me le faire savoir ${ }^{25}$. A la réponse de Millioud jusqu'ici non retrouvée, Pareto réplique le 20 mai 1912: «Je regrette vivement de devoir vous ennuyer encore avec ma prose, après le congé que vous me signifiez; mais votre lettre contient, à mon égard, une imputation que je ne puis paraître accepter, en la passant sous silence ${ }^{26}$. Il se dit certain de «n'avoir jamais manqué en quoi que ce soit, non seulement aux égards que je vous devais comme à un collègue que j'estime et que j'honore, mais encore aux devoirs de l'amitié qui existait entre nous et qui m'était précieuse». Après avoir accepté la thèse, y avoir apporté des corrections linguistiques, "vous m'avez dit que vous trouviez très mauvaise la thèse, qu'elle serait vivement attaquée, que vous n'iriez probablement à la soutenance, ou vous y iriez vers la fin de la séance». Ceci étant, «je ne pouvais pas supposer que l'attaque viendrait de vous ». Le droit et le devoir légitimant la défense, «je voudrais bien savoir comment j'ai pu vous manquer d'égards [...]. Je vous avais d'ailleurs averti que je saurais répondre aux attaques ». "Mais puisque tous mes collègues me tombaient dessus, j'ai dû chercher autre part la bienveillance et l'aide qu'ils me refusaient, et j'ai été heureux de les trouver chez Monsieur le docteur Boven, qui n'avait pas entièrement oublié son professeur, vieux, seul, et malade. [...] En résumé, je crois que je n'ai, moi, absolument rien dit contre vous; je me suis borné à me défendre, comme l'animal qui, pour cela, était dit très méchant. N'ayant rien fait ni dit contre vous, je n'ai rien à me reprocher».

L'attitude de Millioud consterne Pareto: «Dieu! Quelle haine vivace! [...] Mais je ne lui en veux pas; c'est un impulsif qui croit être persécuté par des gens qui n'ont que de l'amitié pour lui » ${ }^{27}$. Pareto répète n'avoir jamais empiété «le moins du monde sur les attributions » de ses collègues, s'être occupé de la thèse avec le «plein consentement de Millioud [...] il en a approuvé la subsistance, tout en blâmant la forme, surtout la langue en laquelle elle était écrite. Il a même eu l'obligeance de corriger un peu cette langue». Pareto récuse la différence établie entre la thèse et ses théories. «J'ai dit, j'ai répété, je répète que ce sont mes théories que Mademoiselle Kolabinska a exposées, que c'est la méthode employée

${ }^{24}$ Ce document a été publié in G. Busino et P. Bridel, L’Ecole de Lausanne de Léon Walras à Pasquale Boninsegni, Lausanne, Université, 1987, pp. 59-60.

${ }^{25}$ Fondo Vilfredo Pareto della Banca Popolare di Sondrio- IT PopSo FP R 17C303.

${ }^{26}$ Fondo Vilfredo Pareto della Banca Popolare di Sondrio- IT PopSo FP R 17C305. La lettre a été publiée et annotée par Pier Carlo Della Ferrera dans Vilfredo Pareto (1848-1923). L'uomo e lo scienziato. A cura di G. Manca, Milano, Scheiwiller, 2002, pp. 343-345.

27 V. Pareto, O.C., 19, pp. 768-771. 
dans tout mon ouvrage de Sociologie dont elle a fait usage, et que, par conséquent, les critiques adressées à ces théories et à cette méthode, si elles sont fondées, sont également méritées par tout mon Traite de Sociologie.[...] le seul reproche qu'on peut adresser à Mademoiselle Kolabinska est d'avoir transcrit trop littéralement des passages de mes leçons, en sorte que plusieurs personnes ont reconnu mon style dans sa thèse.[...] En conclusion, je n'ai absolument rien dit contre mes collègues. J'ai été attaqué à travers la thèse d'une candidate, et je me suis borné strictement à me défendre, sans attaquer personne. Je crois donc être le seul qui ait eu à souffrir de ces incidents ${ }^{28}$.

Le 20 juillet 1912, Pareto écrit de nouveau à Roguin: «Vous reconnaissez vous-même l'existence de cette animosité, car vous écrivez 'J'ai été fort surpris par l'animation, inattendue pour moi, de Monsieur Millioud'. Cette animation préexistait à l'intervention de Monsieur Boven, qui peut l'avoir accrue, mais qui ne l'a pas produite. Ne trouvez-vous pas que c'est là s'engager dans une voie dangereuse et qui peut faire beaucoup de tort à l'Université ?» ${ }^{29}$

Ayant pris connaissance du procès-verbal de la séance de soutenance du 10 mai 1912 rédigé par Millioud, Pierre Boven, le 13 décembre 1964, a écrit : «Quant à mon opinion sur le procès-verbal dont vous m'envoyez copie, il m'est pénible de dire que ce texte incohérent n'exprime qu'une vérité: l'état d'esprit de ce pauvre M. Millioud, alors dans l'un de ses mauvais moments. Le souci de vous renseigner exactement m'oblige à vous dire que M. Millioud, issu jadis de la Faculté de théologie de Lausanne, était un caractère bizarre, inquiet, parfois 'persécuté' et d'une activité décousue. Pareto ne le connaissait sans doute que comme professeur de philosophie, lorsqu'il le chargea de traduire la Sociologie, édition italienne, et d'en faire une version française. Or, il arriva que M. Millioud prit en aversion une étudiante polonaise dont Pareto 'dirigeait la thèse', comme on dit, Mademoiselle Kolabinska, fille chétive et très timide, qui redoutait l'épreuve finale. Certains propos et le comportement de M. Millioud furent tels que Pareto craignit pour son élève un échec à la soutenance. Ne pouvant assister à ladite soutenance en raison de sa santé, il me demanda si je consentirais à m'y rendre, porteur d'une lettre de sa main, lettre donnant son opinion sur le travail présenté, et si, le cas échéant, je serais disposé à défendre sa manière de voir. J'y consentis volontiers. Vous n'aurez pas de peine à croire que la lettre en question était d'une parfaite correction, exempte par conséquent de tout propos et de toute allusion qui pût raisonnablement offenser qui que ce fût. Comme d'habitude, les experts émirent tour à tour leurs avis, et lorsque ce fut M. Millioud, il fit à la dissertation des critiques dont je n'ai pas gardé un souvenir exact, mais avec cette conclusion que la candidate aurait mieux fait de ne pas entreprendre ce travail. Lorsque la parole fut offerte au public, ai-je été le premier à m'exprimer, ou fut-ce M. Scalfati, ou quelqu'un d'autre, je ne sais plus. Quoi qu'il en soit, je suis certain qu'en accomplissant ma mission je n'ai aucunement failli au respect que je devais à tous les membres de la commission, y compris M. Millioud. Néanmoins celui-ci éclata

${ }^{28}$ Cette lettre à Ernest Roguin, du 14 juillet 1912, a été publiée par P. C. Della Ferrera, in Vilfredo Pareto (1848-1943)..., op. cit., pp. 347-350.

${ }^{29}$ Cette lettre a été publiée et annotée par P. C. Della Ferrera, in Vilfredo Pareto (1848-1943), op. cit., pp. 351-353. 
soudain en violentes protestations, disant que je l'insultais. Je vois et j'entends M. Roguin demandant quel propos blessant j'avais tenu et répétant: 'Je n'ai rien entendu physiquement !... Physiquement je n'ai rien entendu !...' Dès lors, brouhaha, confusion telle que le président déclara: 'Puisque la discussion provoque du trouble, je lève la séance. La commission va entrer en délibération... etc.' Le public se retira donc et n'attendit pas longtemps d'être rappelé, pour entendre proclamer que Mademoiselle Kolabinska obtenait le doctorat....» ${ }^{30}$.

Dans l'impossibilité de rétablir avec Millioud des rapports d'une bonne amitié, que faut-il faire pour la traduction de son Traité de sociologie? Pareto hésite: «[...] malgré la conduite à mon égard de Mr. Millioud, je ne puis oublier que nous avons été bons amis, et je veux avoir pour lui les égards qu'il n'a pas eu pour moi ${ }^{31}$. En charger une autre personne? Mais alors «rien ne lui ferait sortir de la tête que cela était prémédité et que cela c'est l'effet d'un de ces noirs dessins ». Pour cette raison il décide d'attendre trois mois avant de confier la traduction à une autre personne.

La réconciliation avec cet homme au «caractère bizarre, inquiet, parfois 'persécuté' et d'une activité décousue » est désormais impossible. Il ne supporte plus la raillerie et l'insolence de celui qui lui disait: "L'ironie, évidemment, est l'arme de la minorité. La majorité des hommes est faite d'imbéciles et un peu aussi de canailles. Il faut donc la tenir en respect par la force; et dans la période de transition, où cela ne se fait pas, il ne reste qu'à rire des imbéciles et de la canaille.»

Le travail de traduction du Traité est, finalement, confié à Boven ${ }^{32}$. Celui-ci corrige également les épreuves de l'édition italienne tout en travaillant à la version française. La traduction, la vérification, la mise au clair de nombreux passage du texte se prolongent jusqu'aux débuts de $1913^{33}$. Boven trouve l'éditeur disposé à publier le Traité . $^{34}$.

Le travail sur les épreuves en français commence dès le mois de juillet 1913. En juin 1914, Boven est nommé substitut du procureur général du Canton de Vaud. Mobilisé à plusieurs reprises pendant la guerre, il est juge d'instruction pour les affaires économiques du Canton de Vaud ainsi que substitut du procureur général $^{35}$, mais continue à s'occuper de la correction des épreuves, à refaire la traduction de certains passages de l'œuvre, à aider le Maître de Céligny de diffé-

${ }^{30}$ G. Busino et P. Bridel, L'Ecole de Lausanne de Léon Walras à Pasquale Boninsegni, op. cit., 59-66.

31 V. Pareto, O.C., 19, pp. 770-771, lettre du 21 mai 1912. Dans la lettre du 31 mai 1912 (Fondo Vilfredo Pareto della Banca Popolare di Sondrio, IT PopSo FP R17C311) nous lisons: «Il est affecté de la manie de la persécution, et je suis sûr qu'il croit que toute la soutenance de Mademoiselle Kolabinska a été un coup monté contre lui pour servir les noirs dessins de conspirateurs pervers.»

${ }^{32}$ V. Pareto, O.C., 19, p. 779, lettre du $1^{\text {er }}$ août 1912.

${ }^{33}$ V. Pareto, O.C., pp. 786, 789, 790, 793, 794-795, 799, 800, 800-801, 806-807, 810, 810-811, 811-812.

${ }^{34}$ V. Pareto, $O, C$., 19, pp. 813-814, lettre du 14 février 1913: « Je suis bien de votre avis que l'opération doit être traitée au point de vue commercial mais pourtant pas exclusivement, car un ouvrage scientifique de ce genre, il faut éviter toute difficulté avec l'éditeur au sujet des épreuves ». Voir également O.C., 19, pp. 815, 817, 819 .

35 V. Pareto, O. C., 19, p. 990, lettre du 5 octobre 1917. 
rentes manières ${ }^{36}$. Pareto en est si reconnaissant qu'il lui donne la propriété littéraire de la traduction française du Traité $e^{37}$.

En 1915, Millioud assume la direction de la Bibliothèque universelle et Revue suisse, publie des articles dans des quotidiens romands, reprend des textes déjà parus dans le livre La caste dominante allemande: sa formation, son rôle ${ }^{38}$, donne diverses préfaces à des livres sur l'Allemagne et défend des points de vue très critiques sur la politique allemande. En décembre 1915 il est condamné par le Tribunal fédéral pour la publication, dans la Bibliothèque universelle et Revue suisse, d'un article virulent de l'écrivain Paul Stapfer traitant l'Empereur Guillaume II de «bandit couronné» digne de l'«exécution capitale». En 1917, Millioud est nommé président de l'Ecole des Sciences sociales et politiques. Pareto restera muet mais pas indifférent. Il demandera à Roguin d'obtenir que Millioud renonce « volontairement à la mission [répartir ses livres entre les bibliothèques des facultés de sciences humaines] qu'il a de par l'acte de donation de la bibliothèque à l'Université » ${ }^{39}$. S'il «renonce spontanément à la mission que lui donne l'acte de donation de la bibliothèque, je considérerai sa résolution comme un acte de délicatesse, qui bien que sans nuire à une future réconciliation, que je continuerai à désirer, la facilitera. M. Millioud peut motiver son acte de renoncer à cette mission par le travail dont il est surchargé; ce dont on vient d'avoir une preuve récente, et n'a nul besoin de faire mention de ma demande transmise par vous ${ }^{40}$. L'intéressé se borne à écrire à Roguin qu'il accepte d'être remplacé mais par le professeur André Mercier. Pour Pareto, cela n'est pas suffisant. Puisque une lettre ne modifie pas le contrat, Millioud doit respecter la procédure légale. Sa manière d'agir lui paraît analogue à celle eue à l'occasion d'une certaine soutenance de thèse. « M. Millioud pense probablement qu'il pourra plus facilement s'imposer à mes héritiers qu'à moi. Il me faut pourvoir à cette éventualité ${ }^{41}$.

Le conflit mondial préoccupe beaucoup Pareto et rend ses activités d'écrivain plus difficiles et compliquées. Il l'avoue à Boven: «Cette guerre sera un des grand événements de l'histoire du monde», "La neutralité suisse est, ce me semble, parfaitement assurée à l'heure actuelle... Je crois que la guerre sera longue», «cette guerre durera encore au moins trois ans. Espérons au moins qu'elle s'éloigne de la Suisse ${ }^{42}$. La mobilisation touche également Boven. Dès que sa période de service est achevée ${ }^{43}$, il reprend son travail de traduction et la révision des épreuves, tout en faisant face à l'augmentation de ses activités professionnelles. Les dossiers économiques qu'il instruit le font douter de la rationalité des

${ }^{36}$ V. Pareto, O. C., 19, pp. 872 et 879.

37 G.Busino et P. Bridel, op. cit., pp. 62-65.

${ }^{38}$ Lausanne, Payot et Paris, Sirey, 1915.

${ }^{39}$ Lettre du 16 décembre 1915 in Fondo Vilfredo Pareto della Banca Popolare di Sondrio - IT PopSo FP R 19 C007.

${ }^{40}$ Lettre du 18 décembre 1915 in Fondo Vilfredo Pareto della Banca Popolare di Sondrio - IT PopSo FP R 19C010.

${ }^{41}$ Letre du 22 janvier 1916 in Fondo Vilfredo Pareto della Banca Popolare di Sondrio - IT PopSo FP R19C043.

${ }^{42}$ V. Pareto, O. C., 19, pp. 879, 880, 886, 889.

${ }^{43}$ V. Pareto, O. C., 19, p. 890. 
comportements humains et de la possibilité de bien circonscrire les actions économiques. Les bouleversements provoqués par la guerre dans tous les aspects de la société, l'accroissement du rôle de l'Etat, la bureaucratisation de la vie quotidienne, lui paraissent prendre des tournures périlleuses. Pareto le réconforte et lui conseille de réunir tous les matériaux qu'il peut en vue de publier un livre sur le sujet à la fin de la guerre. «Au fond, l'homme est un animal essentiellement religieux, et ce n'est que la forme de cette religion qui est très variable. La haine du mécréant, de l'hérétique demeure, tout en changeant d'objet ${ }^{44}$. En attendant il l'engage à écrire des articles sur les bourses, sur le commerce extérieur, sur la Société suisse de surveillance économique, sur l'ingérence de l'Etat dans la vie économique, sur l'évolution de la consommation et sur les emprunts pour la mobilisation $^{45}$. Dès la fin de la guerre, il se demande s'il «convient maintenir en vigueur les prescriptions économiques édictées pendant la guerre ${ }^{46}$. Il traduit et adapte le manuel d'économie de son ancien ami Robert A. Murray ${ }^{47}$. En même temps, il commence à mettre en forme les matériaux pour le livre Le prix normal. Essai sur la lutte contre les crises économiques et la spéculation illicite, publié en $1924^{48}$, où l'on peut lire: «En résumé, le législateur proscrit comme illicite toute spéculation où il voit un accaparement, et il assimile aux spéculations les actes commerciaux les plus simples, dès qu'il croit y découvrir une manœuvre pour modifier les prix dans le sens redouté (renchérissement ou avilissement). Mais l'appréciation du législateur varie beaucoup sur les dangers que présentent les actes flétris; elle dépend étroitement de l'humeur du public et des passions qui l'agitent. Tour à tour inquiète et sévère ou tranquille et complaisante, elle varie au gré d'impressions passagères, bousculant sans égard les principes les mieux établis, les droits les plus responsables dans sa fuite incessante devant l'ignorance irritée. Ces lois sont presque toujours confuses, vexatoires et d'une sévérité sans mesure; rarement applicables dans leur intégralité, elles frappent de coutume certaines victimes expiatoires désignées par leur confession, leur nationalité, leurs succès, leurs attaches, ou simplement l'antipathie qu'inspire leur personne. [...] durant la dernière guerre, et dans des pays très civilisés, le zèle de certains chevaliers pourfendeurs de mercantis n'était, si je puis dire, que la sublimation d'un antisémitisme agressif. La versatilité du législateur explique très bien l'incohérence des mesures prises contre la spéculation, mais pas suffisamment l'inconséquence d'un droit sévère au petit et au moyen commerce, bienveillant aux vastes coalitions d'accapareurs ». Ces lois contre la spéculation ne relèvent ni du droit ni

${ }^{44}$ V. Pareto, O. C., 19, p. 899, lettre du 27 juillet 1915.

${ }^{45}$ P. Boven, Corrispondenza della Svizzera, «Il corriere economico», n. 7, 13 luglio 1916, pp. 185-186; Ibid., I. n. 9, 27 luglio 1916, pp. 234-235; Ibid., I, n. 11, 10 agosto 1916, pp. 285-286; Le borse al principio della guerra, I, n. 15, 7 settembre 1916, pp. 379-380; La SSS. Sua organizzazione, Ibid., I, n. 20, 12 ottobre 1916, pp. 503-504; Corrispondenza dalla Svizzera, Ibid., II, n. 8, 22 febbraio 1917, p. 146.

${ }^{46}$ Convient-il maintenir en vigueur, après la fin complète de la guerre, les prescriptions économiques contre la spéculation illicite?, in «Actes de la Société suisse des juristes », 1920, fasc. 1, pp. 1-56.

${ }^{47}$ R. A. Murray, Leçon d'économie politique, suivant la doctrine de l'Ecole de Lausanne. Ed. française traduite et adaptée de l'italien par Pierre Boven, Paris, Payot, 1920, 501 pp.

${ }^{48}$ Paris, Payot, 1924, 526 pp. 
de l'économie mais du social: «Après tout, l'ignorance, les passions, les intérêts, sont aussi des facteurs importants». Pour conclure: "Un fatras de sentiments, d'intérêts, de préjugés et d'idéologies abstruses enlève à la plupart des hommes toute compréhension du jeu des prix, surtout quand il s'agit d'un trouble économique préjudiciable à ces hommes ${ }^{49}$.

Le 27 janvier 1916, son état de santé s'étant amélioré, Pareto demande au Conseil d'Etat de donner des cours der sociologie pendant le mois de mai, trois fois par semaine, l'après-midi de quatre à cinq heures. La proposition est acceptée et le Conseil d'Etat forme «des vœux sincères pour que vous puissiez, pendant longtemps encore, consacrer à l'Université une partie de votre temps et de votre activité ». Le cours remporte un succès exceptionnel auprès du public. Pareto en est réconforté et envisage de recommencer, avec l'accord des autorités, l'expérience l'année suivante. Millioud en est ému. Le 19 juin 1916, il écrit au Chef du Département de l'Instruction publique et des cultes: «La situation et les compétences de M. Pareto n'étant nullement définies, je sais par une trop claire expérience à quelles intrigues, à quelle guerre personnelle, je vais être en butte pour avoir maintenu l'indépendance de mon enseignement et refusé de me laisser réduire à une vraie domesticité. J'ai les documents écrits de ces agissements, qui n'ont pas été dirigés contre moi seul, et qui ont, une fois déjà, touché au scandale, lors de la soutenance de thèse de Mademoiselle Kolabinska. M. Pareto a marqué suffisamment ses intentions, et l'équivoque de la situation qui risque d'être créée rend inévitable un de ces conflits d'où tout le monde sort rabaissé, sinon avili. C'est pourquoi je désire quitter l'Ecole des sciences sociales dans l'état florissant où nos efforts l'ont mise avant qu'elle retombe dans celui d'où nous l'avons tirée». Pour mettre un terme à cette affaire, le Département notifie à l'Université: «Pour parer à tout imprévu, il est entendu que le Département précisera les conditions dans lesquelles il admettra une nouvelle série de conférences et, comme il ne s'agira que d'un enseignement temporaire et passager, la matière enseignée ne deviendra pas objet d'examen». Puisque cette prise de position le satisfait, Millioud fait savoir que sa lettre de démission n'a plus d'effet.

Pareto a l'impression que ses rapports avec l'Université ne lui sont plus favorables. Une manifestation en son honneur soulève des réticences et des critiques. Il craint aussi «que des savantes intrigues» y préparent l'expulsion des doctrines de l'Ecole de Lausanne. «Je le regrette pour l'Université, mais, pour ma part, cela m'est assez indifférent. Vous m'avez souvent entendu dire que je n'avais pas le moindre instinct de propagande ${ }^{50}$. Boven l'informe que l'inspirateur de ces intrigues est Millioud, soutenu par le secrétaire général du Département de l'Instruction publique et des cultes. Ceux-ci essaient de bloquer les célébrations jubilaires programmées par l'Université à l'occasion du XXV ${ }^{\mathrm{e}}$ anniversaire de l'enseignement parétien ${ }^{51}$. A son ami Pantaleoni Pareto avoue: «[...] mi adoperai

${ }^{49}$ Op. cit. Les citations dans le texte se trouvent aux pages $541-542,543$ et 545.

${ }^{50}$ V. Pareto, O. C., 19, p. 934, lettre du 23 août 1916. Cette lettre a été aussi publiée in G. Busino et P. Bridel, L'Ecole de Lausanne de Léon Walras à Pasquale Boninsegni, op. cit., p. 65.

${ }^{51}$ V. Pareto, O. C., 19, p. 963, lettre du 11 mai 1917 («Je n'avais aucune idée de l'activité déployée par Mr. Millioud, et, d'après ce que vous me dites, il me semble, qu'il a bien du temps à perdre; mais, je n'en ai pas»). Voir aussi O. C., 19, p. 966, lettre du 25 mai 1917: «Je n'ai pas d'autres 
verso il Governo per farlo nominare incaricato di sociologia. Seppi che alcuni professori volevano attaccare le mie teorie via Kolabinska. Avvertii Millioud che mi sarei difeso. Millioud attaccò la teoria della circolazione delle elite "come se fosse una sciocchezza", e disse: "Io fremo pensando che nelle altre Università si leggeranno tali cose". Essendo malato ho incaricato Boven di difendermi. [...] io non l'ho contro il Millioud, perché mi sento molto superiore a tali pettegolezzi. Quindi, quando il Roguin [...] volle mettere pace fra noi due, per parte mia aderii subito. Chi rifiutò fu il Millioud, che in tali occasioni scrisse, disse il Roguin, con tali ingiurie (dico: ingiurie) contro me che il Roguin dichiarò "di non le potere ripetere". [...] Ma tutto ciò è melma che non ho voglia alcuna di rimescolare ${ }^{52}$.

Pour Pareto, l'acharnement haineux de Millioud à son égard est incompréhensible. «Ce n'est nullement en considération du sieur Millioudd, dont je me soucie comme un poisson d'une pomme, que je préférais rester tranquille, mais simplement parce que tout ce qui est vanité m'est fort indifférent $»^{53}$.

Le Jubilé est enfin organisé. Pareto soupçonne que Boven ne sera pas invité à la cérémonie. Pour cette raison il s'empresse d'écrire au doyen de la Faculté de droit André Mercier : «[...] je désirerais beaucoup que le traducteur de la Sociologie en français, Monsieur le docteur Pierre Boven, fût invité à la cérémonie. Son exclusion, si elle ne résultait pas d'une règle absolument générale, sans exception, serait défavorable pour moi $»^{54}$. Le succès du Jubilé est notable et les présentations des travaux économiques et sociologiques du jubilaire remarquables ${ }^{55}$.

Alors que l'amitié entre le vieux professeur et le juge devient, avec les années, très chaleureuse, la relation avec Millioud se détériore toujours plus. L'article sur la ville-Etat de Fiume et la diffusion des doctrines du démographe Filippo Carli sont perçus par Pareto comme des attaques contre ses propres travaux ${ }^{56}$. Filippo Carli, avec ses livres La ricchezza e la guerra (1915), L'altra guerra (1916), L'equilibrio delle nazioni secondo la demografia applicata (1919), avait élaboré une théorie du social basée sur les mécanismes démographiques, sur la différence entre l'action et la réaction, sur la présence d'énergies capables de dépasser l'adaptation passive et de garantir l'intégration. Ce modèle explicatif des faits sociaux est présenté comme un tableau à multiples entrées, abordable de n'importe quel côté et dont on n'envisage un aspect que pour le mettre en rapport avec

\footnotetext{
renseignements que ceux que vous me donnez au sujet de l'activité du "philosophe", et j'ignore de quoi il s'agit. Je ne tiens nullement au "jubilé"; j'avais même prié mes amis de ne pas s'en occuper [...]. Ce n'est nullement en considération du sieur Millioud, dont je me soucie comme un poisson d'une pomme, que je préférais demeurer tranquille, mais simplement parce que tout ce qui est vanité m'est fort indifférent». Voir aussi V. Pareto, O.C., 30, p. 576 n.1. M. Millioud écrira un article plutôt grincheux: Le Jubilé Pareto, «Nouvelle Revue de Lausanne», 11 juin 1917. Voir pour d'autres informations: G. Busino et P. Bridel, L'Ecole de Lausanne de Léon Walras à Pasquale Boninsegni, op. cit., pp. 65-66.

52 V. Pareto, O. C., 28-3, pp. 208-209, lettre du 4 mai 1917.

${ }^{53}$ V. Pareto, O. C., 19, p. 966, lettre du 25 mai 1917.

54 V. Pareto, O. C., 30, lettre du 26 mai 1917.

55 V. Pareto, O. C., 19, p. 970, surtout les notes 2 et 3.

${ }_{56}$ M. Millioud, Fiume italiana, «Nuova Antologia», $1^{\circ}$ febbraio 1919, pp. 347-350. Millioud fera l'édition française du livre de F. Carli, L'équilibre des nations d'après la demographie appliquée, Paris, Payot, 1923.
} 
les autres. Etablissant une moyenne et notant les oscillations du mouvement par rapport à cette moyenne, on observe des variantes. La comparaison des variantes d'ordres divers décèle les lois des phénomènes sociaux. Ces lois sont des lois d'interdépendance et elles expriment des interactions et des corrélations. Le tout n'est que la totalité des corrélations. Les lois naturelles des faits humains, que l'on découvre empiriquement et par induction, sont des lois d'interaction, de relations réciproques, d'interdépendances. Elles modifient la notion de cause, la succession des faits; elles mettent en évidence l'activité et la spontanéité. Les lois naturelles des faits sociaux sont des énoncés des rapports entre ces mêmes faits; elles ne créent pas les faits. Produits par des causes ultimes qui nous échappent, les rapports n'en subsistent pas moins.

Selon Millioud, à l'origine de la traduction en français du livre L'equilibrio delle nazioni, cette construction théorique de Carli se situe dans la droite ligne de Darwin et de Spencer, alors que la doctrine de Pareto est une entreprise analogue à celle de Comte. Rechercher les instincts fondamentaux et invariables des hommes (les résidus), les classer, expliquer les événements de l'histoire par toutes sortes de combinaisons et d'alternances, ne produit qu'une sorte de psychologie sociale, laquelle soumet les faits aux théories. Une telle tentative est aussi vaine que laborieuse car les faits, si divers et opposés soient-ils, demeurent des constructions théoriques, donc des artefacts. Par contre, la démographie se fonde sur les individus, lesquels sont l'unique réalité sociale. Tout part de l'individu et tout doit y revenir. «Tout ce que l'on définit dans la société en dehors de l'individu, et à plus fort raison contre lui, est fiction, abstraction, néant ».

Le décès, en 1923, de Pareto n'apaisa point la rancœur de Millioud, universitaire au caractère ombrageux, à la personnalité rugueuse, aux convictions profondes, à la ténacité de fer et aux exigences tranchées. Il refusa d'en écrire la nécrologie dans la Tribune de Genève, dont il était le collaborateur depuis 1918. En revanche, Boven trace un émouvant portrait du disparu. Il constate que l'œuvre de Pareto converge avec les résultats «de la plus récente école psychologique moderne: celle de Freud. Il est non moins remarquable que ces deux hommes de génie soient demeurés tout à fait inconnus l'un à l'autre » ${ }^{57}$. Six mois plus tard, il passe en revue les contributions économiques et sociologiques du solitaire de Céligny et conclut: «Habituellement, un tel effort de pensée use quelques générations de savants. Qu'ajouter à cette constatation de fait pour glorifier l'œuvre scientifique de Pareto?» ${ }^{58}$ Après le décès de Millioud, en 1925, l'Université demande à Arnold Reymond, Vaudois, professeur à l'Université de Neuchâtel, d'occuper la chaire de philosophie. Reymond accepte l'enseignement de la philosophie mais refuse celui de la sociologie. Pour cet enseignement, plusieurs noms connus sont avancés. Un journal socialiste écrit: «On parle même de M. Boven, substitut du procureur général, ancien élève de feu Vilfredo Pareto, dont il a traduit plusieurs œuvres. En tout cas, les candidats à la succession de Maurice Millioud ne manquent pas. Dans tous les domaines, on se rue sur les postes à repourvoir, quelle que soit leur importance. On nous dit que c'est M. le professeur

${ }^{57}$ P. Boven, Vilfredo Pareto, «Journal de Genève», 21 août 1923 et 22 août 1923.

58 P. Boven, L'œuvre scientifique de Vilfredo Pareto, «Gazette de Lausanne», 11 février 1924 et 13 février 1924. 
de droit Roguin qui occupera momentanément la chaire de sociologie. M. Roguin est un excellent professeur doublé d'un excellent aristocrate. Mais cela n'a pas d'importance, la sociologie n'est-elle pas encore une science... aristocratique ?» ${ }^{59}$

Le 24 février, sept avocats et députés au Grand Conseil vaudois demandent au Conseil d'Etat que la tradition scientifique de la sociologie de Pareto soit assurée avec la nomination de Pierre Boven. Une telle nomination n'entraînera pas de dépenses nouvelles car Pierre Boven pourrait «continuer son travail au Parquet du Procureur Général tout en se chargeant du cours de sociologie». Au mois de mars ces députés-avocats, reviennent à la charge soutenus par le professeur Antoine Rougier. Me H. Vallotton-Warnery écrit, le 25 mars 1925, à Georges Rigassi, député et rédacteur en chef de la Gazette de Lausanne: «[...] nous tenons beaucoup pour l'Université de Lausanne à ce que la chaire de sociologie soit attribuée à $\mathrm{M}$. Boven et non point à $\mathrm{M}$. Boninsegni. Il nous parait incompréhensible que l'on ne profite pas des bonnes dispositions de M. Boven qui consentirait même à renoncer à tout traitement si le Conseil d'Etat le lui demandait. Je ne vous parle pas de M. Boninsegni: tous ceux qui ont été ses élèves à la Faculté de droit où il enseigne l'Economie politique vous en parleront! Au surplus, cette chaire nous paraît devoir revenir tout naturellement à M. Boven qui est Lausannois d'origine, avocat vaudois, officier et qui nous parait emporter à tous les points de vue le pas sur M. Boninsegni ${ }^{60}$.

Le 8 avril le Conseil d'Etat répond aux pétitionnaires que le nom de M. Boven a retenu l'attention de tous les membres du Gouvernement, lequel «apprécie à leur valeur les mérites de ce juristes. Néanmoins, pour des raisons d'économie, nous avons invité la direction de l'Université à étudier, d'entente avec l'Ecole des sciences sociales et la Faculté de droit, les voies et moyens d'assurer cet enseignement sans frais nouveaux pour l'Etat. Des dispositions dans ce sens étaient déjà prises par le conseil de notre école supérieure, lorsque votre pétition nous est parvenue. Nous nous empressons d'ajouter que la possibilité de faire une place à M. Boven dans l'enseignement supérieur n'est pas exclue, l'arrangement pris par l'Université ayant un caractère provisoire, qui pourra toujours être modifié dans la mesure où la situation financière de l'Etat le permettra». Le 21 avril, en réponse à une autre demande du 9 avril du même député-avocat Vallotton-Warnery, le Conseil d'Etat fait savoir que l'Université a chargé le professeur Boninsegni de deux heures de cours de sociologie par semaine et de deux heures de séminaire par quinzaine. Cependant «l'avenir a été réservé en ce qui concerne la nomination d'un professeur spécial de sociologie». Bien que la question reste ouverte, Vallotton-Warnery décide de présenter une interpellation au Grand Conseil. Le chef du Département de l'Instruction publique et des cultes, Dubuis, au cours d'un entretien, confirme au député que l'enseignement de la sociologie a été confié à

${ }^{59}$ Université. Les successeurs du professeur Millioud, «Le droit du peuple», 26 janvier 1926.

${ }^{60}$ Lettre de Maître H. Vallotton-Warnery à Georges Rigassi du 25 mars 1925. Ce dernier répond, le 25 mars 1025: «A première vue, vos arguments me paraissent être irréfutables et vous pouvez être persuadé que cette question retiendra toute mon attention en ma qualité de membre de la sous-commission chargée d'examiner la gestion du département de l'instruction publique.» Voir Gestion 1924. Département de l'Instruction publique et des cultes. Rapport de la sous-commission. Commissaires: MM. Ernest Compondu (Giez) et Georges Rigassi (Lausanne), pp. 42-43, où l'on trouve un résumé de l'affaire. 
Boninsegni à titre «absolument provisoire», que l'on fera appel à Boven lors d'une réorganisation des enseignements de la Faculté de droit. Rassuré, VallottonWarnery renonce, le 24 avril, « jusqu'à l'automne à l'interpellation que je m'étais proposé tout d'abord de faire lors de la plus prochaine session du Grand Conseil ». Boninsegni enseignera la sociologie jusqu'à 1939.

En 1929, Boven est chargé de préparer la refonte du code de procédure pénale vaudois ${ }^{61}$. En 1932, il est nommé procureur général du Canton de Vaud, fonction à laquelle il réserve désormais toute son énergie. Ses rares loisirs sont consacrés à la Société suisse pour la protection de la nature, au Cercle ornithologique de Lausanne et à la publication du livre Autour de nous. Note d'histoire naturelle. Avec 63 dessins de l'auteur ${ }^{62}$. Les pages de ce livre décrivent, avec finesse et perspicacité, les expériences de l'observateur amoureux et tenace de la nature et des oiseaux dans les forêts et au Parc Bourget.

La documentation, dès 1932, n'est guère copieuse. On a retrouvé le manuscrit d'un discours de Boven prononcé au banquet du Groupe radical le 15 novembre 1932, où il dit: " Doctrine, programme, méthode, il en faut; mais gardons nous d'y chercher la force et la certitude de vaincre. Des doctrines néfastes ont souvent triomphé; des programmes peu intelligents ont été favorisés par la chance, des méthodes perverses ont abouti. C'est dans les sentiments obscures et profonds, dans les réactions spontanées de l'individu que gît le secret de la victoire. C'est la vigueur de sa foi qui allume sa vigilance et arme son courage. Ce sont les instincts virils qui gouvernent le monde, et non les théories savantes. En présence de la guerre acharnée qu'on mène contre tout ce qui nous est le plus cher et le plus sacré, en présence du péril imminent, je me dis: soyons vraiment radicaux ».

A l'occasion du départ à la retraite de Pasquale Boninsegni, en 1939, l'Université décide de séparer l'enseignement de la sociologie de celui de l'économie politique. La candidature de Boven est à nouveau évoquée mais la différence de statut et de salaire est si grande qu'il est impossible pour le procureur général d'accepter la charge professorale. Les rapports de Boven avec l'Université n'en sont pas affectés pour autant. La Faculté de droit lui demande, le 28 octobre 1947, de prononcer une leçon à l'occasion de d'ouverture des cours. Boven y traitera des rapports entre les théories juridiques et la pratique juridictionnelle. Dans cette leçon, l'empreinte parétienne demeure puissante. La pratique précède la théorie. La théorie met en forme les expériences, relie les cas particuliers, indique les relations réciproques, les analyse, les classe. En formulant des généralisations, la théorie facilite la transmission, l'explication et la prévision. Elle donne «une vue d'ensemble qui permet au praticien de ne plus considérer les cas particuliers comme nouveaux et fortuits, mais bien comme connus et prévus ». Contrairement à la théorie, la pratique est très complexe puisqu'elle renvoie à des drames humains, à des sentiments, à des vécus moraux ou religieux, à une conception de

${ }^{61}$ P. Boven, Avant-projet du code de procédure pénale, 5 mars 1931, Lausanne, Impr. Jordan et Blanc, 1931, 100 p. Après son entrée en vigueur il publie une édition annotée du Code de procédure pénale du Canton de Vaud, Lausanne, Payot, 1941, 141 p. Quelques années plus tard il publie une étude sur Les institutions pénitentiaires et postpénal dans le Canton de Vaud, in Der Strafvollzug in der Schweiz, 1963, n. 42, Aarau, H. R. Sauerländer, 1963.

${ }^{62}$ Lausanne, Payot, 1939, 216 p. 
la justice et de l'équité. Selon l'orateur, «pour pratiquer le droit, il faut avoir de la tête et du cœur, sans quoi l'équité, la Justice, on en peut bien saisir quelque chose, mais sans jamais y rien comprendre. Pour saisir, en effet, il suffit de voir clair, mais pour comprendre, il faut encore sentir».

Le $1^{\text {er }}$ juillet 1951, Pierre Boven fait valoir ses droits à la retraite. Le Conseil d'Etat lui exprime sa vive reconnaissance pour l'activité qu'il a déployée, avec courtoisie, fermeté et autorité, tout au long de sa carrière de magistrat. Un chroniqueur judiciaire en salua le départ à la retraite ainsi: «Certes, il est arrivé à M. Boven, durant les trente-cinq années qu'il a passées au ministère public vaudois, de se montrer sévère et de réclamer des châtiments exemplaires pour ceux qui avaient enfreint la loi. Mais surtout, il apporta dans l'exercice de sa charge ce souci de la justice, cette minutieuse équité, cette profonde humanité qui caractérisent toute sa personne. Les innombrables délinquants peuvent en témoigner, qu'après les avoir fait condamner, il a suivi, aidé, conseillé dans leur difficile retour vers le droit chemin ${ }^{63}$.

Avec le décès de Pierre Boven, en 1968, disparaît le seul véritable élève de Pareto en Pays de Vaud. Ce décès consacre l'effacement définitif d'une riche mémoire et ouvre la voie à la recherche historique. Ramenant d'autre part à la vie un passé disparu à jamais, la recherche historique nous remet sous les yeux la dramaturgie de la vie universitaire, ses débats tendus, virulents, parfois dérisoires, les jalousies entre collègues, mais aussi les dépits, les envies, les ombrages qui se soldent, sans faillir, par l'oubli le plus total, par la stérilité des idées, des débats scientifiques, des processus culturels, par la désertification de ces champs intellectuels, incalculables et délaissés souvent par le conformisme sclérosant de la doxa académique. Et pour le lecteur d'aujourd'hui, la constatation que la futilité joue un rôle important dans la vie universitaire, qu'à l'Université de Lausanne les principes fondateurs des théories de Walras et de Pareto, les méthodes qu'ils ont enseignées, n'ont été repris par personnes et ni même contestés ou critiqués. Ils ont été simplement couverts par le voile de l'oubli pendant fort longtemps.

${ }^{63}$ Colette Muret, Monsieur le Procureur, «Gazette de Lausanne», 8 avril 1951. Voir également l'article non signé Le procureur général Pierre Boven se retire, «Nouvelle revue de Lausanne», 5 avril 1951. 